\title{
A COUNTEREXAMPLE IN THE THEORY OF DERIVATIONS
}

\section{by FENG WENYING and JI GUOXING}

(Received 18 November, 1987)

Let $B(H)$ be the algebra of all bounded linear operators on a separable, infinite dimensional complex Hilbert space $H$. Let $C_{2}$ and $C_{1}$ denote respectively, the HilbertSchmidt class and the trace class operators in $B(H)$. It is known that $C_{2}$ and $C_{1}$ are two-sided ${ }^{*}$-ideals in $B(H)$ and $C_{2}$ is a Hilbert space with respect to the inner product

$$
(X, Y)=\operatorname{tr}\left(Y^{*} X\right) \quad\left(X, Y \in C_{2}\right),
$$

(where tr denotes the trace). For any Hilbert-Schmidt operator $X$ let $\|X\|_{2}=(X, X)^{1 / 2}$ be the Hilbert-Schmidt norm of $X$.

For fixed $A \in B(H)$ let $\delta_{A}$ be the operator on $B(H)$ defined by

$$
\delta_{A}(X)=A X-X A \quad(X \in B(H)) .
$$

Operators of the form (1) are called inner derivations and they (as well as their restrictions $\left.\delta_{A}\right|_{C_{2}}$ ) have been extensively studied (for example [1-3]). In [1], Fuad Kittaneh proved the following result.

THEOREM $K$. If $A$ is a cyclic subnormal operator and $S \in C_{2}$ is an operator such that $A S=S A$, then for all $X \in B(H)$ we have

$$
\|A X-X A+S\|_{2}^{2}=\|A X-X A\|_{2}^{2}+\|S\|_{2}^{2} .
$$

Hence the range of $\delta_{A} \mid C_{2}$ is orthogonal to the null space of $\left.\delta_{A}\right|_{C_{2}}$ in the usual Hilbert space sense.

In this paper, we give an example which provides an affirmative answer to the following question in [1].

Question 1. Is it necessary to assume $A$ cyclic in Theorem $K$ ?

First we prove a lemma.

Lemma. If $A$ is an operator in $B(H)$ and $S \in C_{2}$ with $A S=S A$, and for all $X \in B(H)$.

$$
\|A X-X A+S\|_{2}^{2}=\|A X-X A\|_{2}^{2}+\|S\|_{2}^{2},
$$

then $A S^{*}=S^{*} A$.

Proof. $\left.\delta_{A}\right|_{C_{2}}$ is a bounded linear operator acting on the Hilbert space $C_{2}$ and $\left(\delta_{A} \mid C_{2}\right)^{*}=\left.\delta_{A} \cdot\right|_{C_{2}}$. Noting that $\left.\overline{R\left(\delta_{A} \mid C_{2}\right)}\right)^{\perp}=N\left(\left.\delta_{A} \cdot\right|_{C_{2}}\right)$, we have $N\left(\left.\delta_{A}\right|_{C_{2}}\right) \subset N\left(\left.\delta_{A} \cdot\right|_{C_{2}}\right)$; therefore $A S^{*}=S^{*} A$. (Here $R\left(\left.\delta_{A}\right|_{C_{2}}\right)$ and $N\left(\left.\delta_{A}\right|_{C_{2}}\right)$ denote respectively the range of $\left.\delta_{A}\right|_{C_{2}}$ and the null space of $\delta_{A} \mid C_{2}$ ).

We give an example showing that $A$ is necessarily cyclic in Theorem $K$.

Glasgow Math. J. 31 (1989) 161-163. 
EXAMPLE. Let $\left\{e_{n}\right\}_{n=-\infty}^{+\infty}$ be an orthonormal basis for a Hilbert space $H$ and let $\left\{c_{n}\right\}_{n=-\infty}^{+\infty}$ and $\left\{d_{n}\right\}_{n=-\infty}^{+\infty}$ be bounded sequences of positive numbers as follows:

$$
\begin{aligned}
& c_{n}= \begin{cases}\frac{n+1}{n+2} \quad(n \geqslant 0), \\
\frac{1}{2-n} \quad(n<0) ;\end{cases} \\
& d_{n}= \begin{cases}c_{n-1}+\frac{1}{2(n+2)(n+1)}=\frac{2 n^{2}+4 n+1}{2(n+2)(n+1)} & (n \geqslant 1), \\
c_{n-1}+\frac{1}{2(3-n)(2-n)}=\frac{5-2 n}{2(3-n)(2-n)} & (n \leqslant 0) ;\end{cases}
\end{aligned}
$$

then for each integer $n, c_{n+1}<d_{n}<c_{n}$. Let $P_{1}$ and $P_{2}$ be operators in $B(H)$ defined by $P_{1} e_{n}=c_{n} e_{n}$ and $P_{2} e_{n}=d_{n} e_{n}$, for each integer $n$. Let $\hat{H}=\sum_{n=1}^{+\infty} \oplus H_{n}, \hat{P}_{1}=\sum_{n=1}^{+\infty} \oplus P_{n}$ and $\hat{P}_{2}=\sum_{n=1}^{+\infty} \oplus P_{n}^{\prime}$, where for each $n, H_{n}=H, P_{n}=P_{1}$ and $P_{n}^{\prime}=P_{2}$. Let $V_{H}^{n=1}$ denote the unilateral shift on $\hat{H}$, i.e., $V_{H}\left(x_{1}, x_{2}, \ldots\right)=\left(0, x_{1}, x_{2}, \ldots\right)$ for each $\left(x_{1}, x_{2}, \ldots\right)$ in $\hat{H}$. Let $T_{1}=V_{H} \hat{P}_{1}$ and $T_{2}=V_{H} \hat{P}_{2}$ on $\hat{H}$. The operators $T_{1}$ and $T_{2}$ are pure quasinormal operators by [4], and so they are pure subnormal operators.

For each positive integer $m$ define $X_{m}$ and $Y_{m}$ in $B(H)$ by

$$
X_{m} e_{n}=\frac{1}{2^{|n|}}\left(\frac{d_{n}}{c_{n}}\right)^{m} e_{n}
$$

and

$$
Y_{m} e_{n}=\frac{1}{2^{|n|}}\left(\frac{c_{n-1}}{d_{n}}\right)^{m} e_{n-1},
$$

for $n=\ldots-2,-1,0,1,2, \ldots$ Observe that $X_{m}$ and $Y_{m}$ are compact $(m=1,2, \ldots)$, that $\left\|X_{m}\right\| \rightarrow 0$ and $\left\|Y_{m}\right\| \rightarrow 0$. Also $X_{m} P_{1}=P_{2} X_{m-1}, Y_{m} P_{2}=P_{1} Y_{m-1},(m=2,3, \ldots)$.

Let $X=\sum_{m=1}^{+\infty} \oplus X_{m}$ and $Y=\sum_{m=1}^{+\infty} \oplus Y_{m}$ on $\hat{H}$. Thus $X$ and $Y$ are compact, $X T_{1}=T_{2} X$, $T_{1} Y=Y T_{2}$; hence $(Y X) T_{1}=Y T_{2} X=T_{1}(Y X)$.

Next, we shall prove that $X$ is a Hilbert-Schmidt operator.

Let $v_{i j}$ have $e_{i}$ in the $j$ th position, zeros elsewhere for $j=1,2, \ldots$ and $i=\ldots,-2$, $-1,0,1,2, \ldots ;$ thus $\left\{v_{i j}\right\}$ is an orthonormal basis for the Hilbert space $\hat{H}$. From the definition of $X$, we have

$$
X v_{i j}=\left(\ldots, 0, \ldots, 0, \frac{1}{2^{|i|}}\left(\frac{d_{j}}{c_{i}}\right)^{j} e_{i}, 0, \ldots\right)
$$




$$
\begin{aligned}
\left\|X v_{i j}\right\| & =\frac{1}{2^{|i|}\left(\frac{d_{i}}{c_{i}}\right)^{j}} \\
\sum_{i, j}\left\|X v_{i j}\right\| & =\sum_{i=-\infty}^{+\infty} \sum_{j=1}^{+\infty} \frac{1}{2^{|i|}\left(\frac{d_{i}}{c_{i}}\right)^{j}} \\
& =\sum_{i=-\infty}^{+\infty} \frac{1}{2^{|i|}} \frac{d_{i}}{c_{i}-d_{i}} \\
& =\sum_{i=-\infty}^{0} \frac{1}{2^{|i|}} \frac{d_{i}}{c_{i}-d_{i}}+\sum_{i=1}^{+\infty} \frac{1}{2^{i}} \frac{d_{i}}{c_{i}-d_{i}} \\
& =\sum_{i=0}^{+\infty} \frac{1}{2^{i}} \frac{d_{-i}}{c_{-i}-d_{-i}}+\sum_{i=1}^{+\infty} \frac{1}{2^{i}} \frac{d_{i}}{c_{i}-d_{i}} \\
& =\sum_{i=0}^{+\infty} \frac{2 i+5}{2^{i}}+\sum_{i=1}^{+\infty} \frac{2 i^{2}+4 i+1}{2^{i}}<+\infty .
\end{aligned}
$$

Thus $X$ is a Hilbert-Schmidt operator, and so is $Y X$. Note that the operator $Y X$ is compact and $(Y X) T_{1}=T_{1}(Y X)$, but $T_{1}$ is a pure subnormal operator; therefore $(Y X)^{*} T_{1} \neq T_{1}(Y X)^{*}$. From the lemma, we know that Theorem $K$ does not hold for $T_{1}$; thus if Theorem $K$ holds, then $A$ must by cyclic.

The example above gives an affirmative answer to Question 1.

\section{REFERENCES}

1. Fuad Kittaneh, On normal derivations of Hilbert-Schmidt type, Glasgow Math. J. 29 (1987), 245-248.

2. J. H. Anderson, On normal derivations, Proc. Amer. Math. Soc. 38 (1973), 135-140.

3. L. A. Fialkow, A note on norm ideals and the operator $X \rightarrow A X-X B$, Israel J. Math. 32, (1979), 331-348. $127-139$.

4. L. R. Williams, Quasisimilarity and hyponormal operators, J. Operator Theory 5 (1981),

DepartMent of Mathematics

SHAANXI NORMAL UNIVERSITY

X'AN

People's Republic of China 